\title{
Incidência de Lúpus Eritematoso Sistêmico em Natal, RN - Brasil(*)
}

\section{Incidence of Systemic Lupus Erythematosus in Natal, RN, Brazil}

\author{
Maria José Pereira Vilar ${ }^{(1)}$, Juliana Martins Rodrigues ${ }^{(2)}$ e Emilia Inoue Sato ${ }^{(3)}$
}

\section{RESUMO}

Objetivo: estudar a incidência de lúpus eritematoso sistêmico (LES) na cidade de Natal, RN, Brasil. Métodos: foram incluídos somente pacientes residentes em Natal, RN, com idade $\geq 15$ anos, com pelo menos, quatro critérios do ACR, entre $1 / 1$ / 2000 e 31/12/2000, excluindo lúpus droga-induzido. Foram utilizadas quatro fontes para identificação de pacientes: 1) hospital universitário; 2) postos de saúde de hospitais da rede pública; 3) especialistas (reumatologistas, dermatologistas, nefrologistas e hematologistas) em clínicas e hospitais privados e 4) resultados positivos de FAN $(\geq 1: 80)$ de três principais laboratórios da cidade. Médicos foram informados, por cartas, dos procedimentos para notificação. Dados do Censo 2000 foram utilizados para o cálculo das taxas de incidência. Standardized Mortality Ratio (SMR) foi aplicado para comparação das taxas de incidência. O valor de $\mathrm{p}<0,05$ foi considerado significativo. Resultados: foram identificados 43 novos casos de LES, com uma incidência calculada de 8,7 por $100.000 /$ ano (95\% IC 6,3-11,7/100.000). Trinta e oito mulheres 14,1:100.000/ano (95\% IC 10,0-19,3) e 5 homens 2,2 por 100.000/ano (95\% IC 0,7-5,2). A média de idade dos pacientes foi de 31,8 anos (95\% IC 27,8-35,8), sendo de 31,4 anos (95\% CI 27,2-34,7) nas mulheres e de 35 anos (95\% CI 27,8-52,2) nos homens. A mediana de duração da doença (tempo entre a primeira manifestação clínica de LES e o diagnóstico ) foi de 10 meses (1 a 72 meses). Conclusões: a incidência de LES em Natal, RN, parece ser maior que a relatada em outras regiões do mundo. Fatores étnicos e ambientais, como maior exposição à radiação ultravioleta, parecem contribuir para essas diferenças observadas.

Palavras-chave: lúpus eritematoso sistêmico, incidência, Brasil, epidemiologia.

\begin{abstract}
Objective: To study the incidence of Systemic Lupus Erythematosus (SLE) in the city of Natal, Brazil. Methods: Only patients living in the city of Natal, older than 15 years old, who fulfilled at least 4 of ACR criteria between January 1st, 2000 and December 31 $1^{\text {st }}$, 2000, were included. Four sources were used to identify new cases of SLE: 1) the University Hospital; 2) "health units" and hospitals of the public health network; 3) specialists at private hospitals and outpatient clinics; 4) three laboratories performing antinuclear antibody (ANA) determination. Physicians were contacted by mail, receiving explanations about the study and directions on how to collaborate. Brazilian population census data (2000) was used to calculate incidence rate. The Standardized Mortality Ratio (SMR) was used to compare the incidence rates. The 95\% confidence intervals (95\% CI) were calculated and a $P$ value $<0.05$ was considered statistically significant. Results: Forty three patients were diagnosed as SLE new cases in 2000. The calculated incidence was 8.7/100 000/year (95\% CI 6.3-11.7). Thirty eight patients were female 14.1/100.000/year (95\% CI 10.0-19.3) and 5 were male 2.2/100.000/year (95\% CI $0.7-5.2)$. The mean age of SLE new cases was 31.8 years old (95\% CI 27.8-35.8). The mean age for women was 31.4 years old and for men it was 35.0 years old. The median of disease duration (time between onset of the first ACR criterion for SLE and diagnosis) was 10 months (1-72 months). Conclusions: The incidence of SLE in Natal is apparently higher than those reported in other regions of the world. The observed differences may be due to ethnical and/or environmental factors.
\end{abstract}

Keywords: systemic lupus erythematosus, incidence, Brazil, epidemiology.

\footnotetext{
* Estudo realizado pelo Projeto Pronuclear, com o apoio do Fundo de Incentivo à Pesquisa e Ensino da Sociedade Brasileira de Reumatologia. Recebido em 24/02/03. Aprovado, após revisão, em 28/07/03.

1. Professora Adjunta do Departamento de Medicina Clínica da Universidade Federal do Rio Grande do Norte - UFRN.

2. Médica Residente em Clínica Médica do Hospital Universitário Onofre Lopes - UFRN.

3. Professora Titular da Disciplina de Reumatologia da Universidade Federal de São Paulo - UNIFESP.

Endereço para correspondência: Maria José Pereira Vilar. Rua Jundiaí, 702, Tirol, Natal-RN. E-mail: vilarmj@ufrnet.br
} 


\section{INTRODUÇÃO}

Estudos epidemiológicos sobre prevalência e incidência de lúpus eritematoso sistêmico (LES) apresentam uma grande variação nos resultados entre as várias populações estudadas no mundo ${ }^{(1-4)}$, mesmo dentro de um mesmo país, mostrando uma taxa anual de incidência de LES que varia de 1,8 a 7,6 casos por 100.000 habitantes/ano ${ }^{(5-8)}$. Alguns aspectos, como a seleção de pacientes, principalmente em relação aos diferentes critérios de classificação da doença, parecem contribuir para as diferenças nos resultados encontrados em vários estudos $^{(3,7,9,10)}$. Fatores genéticos e ambientais, como a exposição à luz ultravioleta e a maior utilização de hormônios, parecem estar implicados na expressão e no aparecimento da doença ${ }^{(11-14)}$.

No Brasil, poucos estudos epidemiológicos são realizados na área da reumatologia, entre os quais podem-se citar os estudos da prevalência de artrite reumatóide em amostras da população brasileira ${ }^{(15)}$. Em relação ao LES, a maioria dos estudos foi realizada nos Estados Unidos e na Europa, sendo este o primeiro estudo de incidência da doença no Brasil e em região de clima tropical, à exceção de um estudo realizado na Ilha de Curaçau ${ }^{(3)}$.

Partindo do princípio que fatores ambientais, como as condições socioeconômicas e climáticas, possam estar implicados no aparecimento e nas manifestações clínicas do LES e em razão da escassez de estudos que abordem este aspecto em nosso país, parece interessante um estudo da incidência da doença no nordeste do Brasil, região de clima tropical e com características peculiares. O objetivo deste trabalho, portanto, é estudar a incidência de LES na cidade de Natal, RN-Brasil, no período de 1/1/2000 a 31/12/2000.

\section{PACIENTES E MÉTODOS}

\section{POPULAÇÃo e Área do Estudo}

A origem dos dados da população estudada foi baseada no Censo 2000 da população brasileira do IBGE (Instituto Brasileiro de Geografia e Estatística) ${ }^{(16)}$. A população de Natal é de 709.422 habitantes (376.726 mulheres e 332.696 homens), sendo 493.239 habitantes (269.900 mulheres e 223.339 homens) com idade maior ou igual a 15 anos. Em relação à raça e à cor, os pacientes usaram a auto-referência, sendo classificados como branco, preto, pardo, amarelo ou índio, de acordo com o IBGE.

Natal é uma cidade litorânea, de clima tropical, com uma temperatura média anual em torno de 27 graus.
Segundo a Estação Meteorológica de Natal, RN, a quantidade de luz solar na área varia entre 205 e 288 horas/mês. O índice ultravioleta (Índice UV), que varia em escala de 0 a $10+{ }^{(17)}$, de acordo com o Instituto de Pesquisas Espaciais de São José dos Campos (INPE), SP, é considerado alto em Natal, sendo maior que $7+$ na maior parte do ano ${ }^{(18)}$.

\section{SELEÇÃO dE PACIENTES}

Foram incluídos no estudo somente indivíduos residentes na cidade de Natal, com idade maior ou igual a 15 anos. Foram definidos como casos incidentes de LES aqueles pacientes que preencheram, pelo menos, quatro critérios da classificação do Colégio Americano de Reumatologia (ACR) para $\mathrm{LES}^{(19)}$, dentro do prazo definido para o estudo, ou seja, de 1/1/2000 a 31/12/2000. Foi critério de exclusão o lúpus induzido por drogas. A duração da doença foi definida como o tempo entre o início dos sintomas atribuído pelo médico à doença e o diagnóstico. A incidência foi estimada dividindo o número de casos novos encontrados pela população de Natal com idade maior ou igual a 15 anos de acordo com o Censo 2000. O protocolo básico de notificação foi composto de dados demográficos como sexo, idade, data de nascimento, cor e dados clínicos como duração da doença e critérios de classificação do ACR.

Quatro fontes distintas foram utilizadas para a identificação de pacientes: 1) Hospital Universitário da Universidade Federal; 2) hospitais e postos de saúde das redes públicas municipal e estadual; 3) clínicas e hospitais privados; 4) três principais laboratórios da cidade que realizam a pesquisa de fator antinuclear (FAN).

Antes do início do estudo e por mais duas vezes subseqüentes, todos os médicos residentes em Natal foram informados, por cartas, dos objetivos do estudo e do procedimento para notificação dos pacientes. Concomitantemente, cartazes de esclarecimento sobre a notificação foram colocados nos hospitais e principais unidades de saúde da rede pública.

Os médicos da rede pública foram requisitados a encaminhar estes casos ao hospital universitário para notificação (aqueles já diagnosticados) e para confirmação diagnóstica (os casos suspeitos) pelo reumatologista (investigador principal). Este último procedimento justifica-se pela dificuldade da realização de exame de auto-anticorpos na rede pública de saúde.

Médicos reumatologistas, dermatologistas, hematologistas e nefrologistas em suas clínicas privadas foram requisitados a notificar, por meio de um formulário simples, os casos 
novos ou casos suspeitos de LES identificados no período do estudo, sendo periodicamente contatados por telefone ou pessoalmente. Os pacientes notificados pelos especialistas em clínica e hospitais privados também foram reavaliados pelo reumatologista (investigador principal) para confirmação diagnóstica.

Durante o ano de 2000, todos os resultados positivos de FAN $(>1: 80)$ realizados no setor de imunologia em três laboratórios de Natal $(\mathrm{n}=340)$ foram notificados e os respectivos médicos requisitantes contatados pelo investigador principal à procura de novos casos.

\section{ANÁLISE ESTATÍSTICA}

Dados do Censo 2000 do IBGE sobre a população brasileira ${ }^{(16)}$ foram utilizados para calcular a taxa de incidência. A população de Natal com idade maior ou igual a 15 anos foi calculada usando os dados do Censo de $1991^{(20)}$ e da Contagem Populacional de $1996^{(21)}$, pois estes dados ainda não estão disponíveis no Censo 2000. Taxa de mortalidade normalizada (SMR - Standardized mortality ratio) foi utilizada para atenuar as diferenças dentro das populações estudadas na comparação das taxas de incidência. Foi calculado o intervalo de confiança 95\% (95\% IC). Valor de $\mathrm{p}<0,05$ foi considerado estatisticamente significante.

\section{RESULTADOS}

Foram encontrados 43 novos pacientes com LES de acordo com os critérios de classificação do $\mathrm{ACR}^{\left({ }^{19)}\right.}$ na cidade de Natal no ano 2000, com uma taxa de incidência de 8,7 por 100.000/ano (95\% IC 6,3-11,7:100.000/ano), sendo 38 mulheres e 5 homens, com incidência de 14,1 por 100.000 /ano (95\% IC $10,0-19,3$ ) e 2,2 por $100.000 /$ ano (95\% IC 0,7-5,2/100.000/ano), respectivamente (Tabela 1).

A média de idade dos pacientes na época do diagnóstico foi de 31,8 anos (95\% IC 27,8-35,8), sendo de 31,4 anos (95\% IC 27,2-35,7) nas mulheres e de 35 anos (95\% IC 17,8-52,2) nos homens (Tabela 2).

TABELA 1

INCIDÊNCIA DE LES EM NATAL, RN - BRASIL, DE ACORDO COM O SEXO, NO PERÍODO DE 1/1/2000 A 31/12/2000

\begin{tabular}{lcccc}
\hline & Número & População & $\begin{array}{c}\text { Incidência } \\
(100.000 / \text { ano })\end{array}$ & $95 \%$ IC \\
\hline Mulheres & 38 & 269.900 & 14,1 & $10,0-19,3$ \\
Homens & 5 & 223.339 & 2,2 & $0,7-5,2$ \\
Total & 43 & 493.239 & 8,7 & $6,3-11,7$ \\
\hline
\end{tabular}

TABELA 2

MÉdia de IDAde (ANOS) dOS 43 NOVOS CASOS DE LES OCORRIDOS EM NATAL, RN - BRASIL, NO PERÍODO DE 1/1/2000 A 31/12/2000

\begin{tabular}{lcccc}
\hline & Número & Média & $95 \%$ IC & Mín - Máx \\
\hline Mulheres & 38 & 31,4 & $27,2-35,7$ & $15,0-72,0$ \\
Homens & 8 & 35,0 & $27,8-52,2$ & $24,0-59,0$ \\
Total & 43 & 31,8 & $27,8-35,8$ & $15,0-72,0$ \\
\hline
\end{tabular}

Trinta e três $(77 \%)$ dos 43 pacientes eram brancos - 28 mulheres e 5 homens - e 10 (27\%) não-brancos - 6 pretos $(14 \%)$ e 4 pardos (9\%).

O pico de incidência de 32,7 por $100.000 /$ ano (95\% IC 15,0-62,1) foi observado em mulheres entre 35 e 39 anos. A taxa de incidência foi maior em mulheres em todas as faixas etárias, exceto entre a faixa de 55-59 anos, com um caso entre homens e nenhum caso em mulheres.

A mediana de duração da doença (tempo entre o início do primeiro critério do ACR e o diagnóstico) foi de 10 meses (1 a 72 meses).

Os 43 novos casos de LES foram identificados a partir das quatro fontes utilizadas: Hospital Universitário (15 pacientes), outros hospitais da rede pública de saúde (5 pacientes), especialistas em clínicas privadas (20 pacientes) e por resultado positivo de FAN nos laboratórios pesquisados (3 pacientes). Os casos foram identificados pelos seguintes especialistas: reumatologistas (33 pacientes), nefrologistas (3 pacientes), dermatologistas ( 2 pacientes), hematologista (1 paciente), cardiologista (1 paciente). Os outros três pacientes foram identificados pelo rastreamento do FAN nos laboratórios. Vinte e nove novos casos $(67,4 \%)$ foram notificados por mais de uma fonte.

\section{DISCUSSÃO}

A maioria dos estudos sobre incidência de LES, realizados nos Estados Unidos, países nórdicos e Reino Unido (Tabela 3), mostra uma preponderância do sexo feminino. Em nosso estudo, o primeiro realizado na população brasileira, encontramos uma taxa de incidência de 8,7 por 100.000/ano, também com uma maior frequiência nas mulheres (38 mulheres e 5 homens). A doença foi mais freqüente entre os pacientes que se auto-referenciaram brancos ( $77 \%$ brancos e $23 \%$ não-brancos). Importante salientar a dificuldade que existe em classificar a população brasileira quanto à raça ou cor, em virtude da grande diversidade de misturas de raças e, ainda, por questões sociais, que podem influenciar na auto-referência que, nem sempre, corresponde à realidade. A maioria da população brasileira, 
incluindo a população de Natal, é descendente da miscigenação entre europeus, negros e índios ${ }^{(25,26)}$.

\section{TABELA 3}

ESTUDOS DE INCIDÊNCIA DE LÚPUS ERITEMATOSO SISTÊMICO (POR 100.000 HABITANTES/ANO) EM VÁRIAS REGIÕES DO MUNDO

\begin{tabular}{|c|c|c|c|}
\hline $\begin{array}{l}\text { Autor, } \\
\text { local do estudo }\end{array}$ & $\begin{array}{l}\text { Total de casos } \\
\text { (n) }\end{array}$ & $\begin{array}{l}\text { Período do } \\
\text { estudo }\end{array}$ & $\begin{array}{c}\text { Incidência } \\
{[95 \% \mathrm{IC}]}\end{array}$ \\
\hline $\begin{array}{l}\text { Fessel }^{(5)} \\
\text { San Francisco (CA) }\end{array}$ & 74 & $1965-1973$ & 7,6 \\
\hline $\begin{array}{l}\text { Nossent*(3) } \\
\text { Curaçao Island }\end{array}$ & 94 & 1980-1989 & 4,6 \\
\hline $\begin{array}{l}\text { Michet et al. }{ }^{(6)} \\
\text { Rochester, MN }\end{array}$ & 25 & 1950-1979 & $1,9[1,2-2,6]$ \\
\hline $\begin{array}{l}\text { Hochberg }^{(8)} \\
\text { Baltimore, MD }\end{array}$ & 302 & 1970-1977 & 4,6 \\
\hline $\begin{array}{l}\text { Uramoto et al.(22) } \\
\text { Rochester, MN }\end{array}$ & 48 & 1980-1992 & $5,5[3,9-7,1]$ \\
\hline $\begin{array}{l}\text { McCarty et al. }{ }^{(7)} \\
\text { Allegheny County, PA }\end{array}$ & 191 & $1985-1990$ & $2,8[2,6-3,2]$ \\
\hline $\begin{array}{l}\text { Jonsson et al. }{ }^{(23)} \\
\text { Southern Sweden }\end{array}$ & 39 & 1981-1986 & $4,0[1,6-6,4]$ \\
\hline $\begin{array}{l}\text { Stalh-Hallengren et al. }{ }^{(24)} \\
\text { Southern Sweden }\end{array}$ & 41 & 1987-1991 & 4,8 \\
\hline $\begin{array}{l}\text { Hopkinson et al. ***(4) } \\
\text { Nottingham, UK }\end{array}$ & 23 & $1989-1990$ & 3,7 \\
\hline $\begin{array}{l}\text { Johnson et al. }{ }^{* *(27)} \\
\text { Birmingham, UK }\end{array}$ & 33 & 1991 & $3,8[2,5-5,1]$ \\
\hline $\begin{array}{l}\text { Vilar, Rodrigues e } \\
\text { SatoNatal-RN- Brasil }\end{array}$ & 43 & 2000 & $8,7[6,3-11,7]$ \\
\hline
\end{tabular}

Diferenças de ordem genética, assim como na metodologia empregada nos vários estudos de incidência de LES no mundo, tornam dificil a comparação entre eles. Uramoto et al. ${ }^{(22)}$ em Rochester, Minnesota, observaram que a incidência de LES triplicou nas últimas quatro décadas nesta região. Por outro lado, Stalh-Hallengren et al. ${ }^{(24)}$, em recente estudo na Suécia, comparando dois estudos de coorte, concluíram que a incidência de LES foi constante durante 11 anos (1981-1991).

Em nosso estudo, a taxa de incidência de LES de 8,7 por 100.000/ano foi, pelo menos, duas vezes maior do que a observada em outros estudos, de acordo com método SMR calculado. Esta incidência foi maior do que a encontrada por Johnson et al. ${ }^{(27)} \mathrm{em}$ Birmingham, Inglaterra $[\mathrm{SMR}=2,2$ $(\mathrm{p}<0,001)]$ onde a prevalência foi de 3,8 por $100.000 /$ ano em 1991. Nossa incidência também foi maior do que a encontrada por Hopkinson et al. ${ }^{(4)}$, em Nottingham, Reino Unido $[\mathrm{SMR}=2,2(\mathrm{p}<0,001)]$, onde foi de 3,7 por 100.000/ano. Na Ilha de Curaçau, Nossent ${ }^{(3)}$ encontrou uma prevalência de 4,6 por 100.000/ano, também menor do que a nossa incidência $[\mathrm{SMR}=1,8(\mathrm{p}<0,001)]$.

No estudo da Ilha de Curaçau $^{(3)}$, a incidência de LES foi semelhante à encontrado por Jonsson et al. ${ }^{(28)}$ na Suécia, ambas menores do que as do estudo de Hochberg ${ }^{(8)}$ em Baltimore, com uma população predominantemente de indivíduos negros. Neste último estudo é sugerido que fatores ambientais e não só fatores genéticos têm influência na expressão da doença, já que esta população é oriunda de uma região da África Central com uma prevalência presumivelmente baixa de LES. A taxa de incidência de 7,6 por 100.000/ano relatada por Fessel ${ }^{(5)}$, em São Francisco, é difícil de ser comparada com outros estudos porque o autor usou para inclusão os critérios anteriores aos critérios revisados do ACR de 1982.

À exceção do estudo realizado na Ilha de Curaçau ${ }^{(3)}$, nenhum estudo sobre incidência de LES foi feito em região de clima tropical. O alto índice UV em Natal (>7 na escala de 0-10+), associado à baixa condição socioeconômica e pouco cuidado com a exposição à luz solar, pode ser um dos fatores que justificam a alta incidência de LES em nosso estudo.

A média de idade de 31,8 anos dos 43 novos casos de LES foi menor que a relatada por Uramoto et al. ${ }^{(22)}$, em Rochester, de 49,2 anos. Nossa média de idade também foi menor do que a encontrada na Suécia ${ }^{(23)}$, com uma média de 50,1 anos. Por outro lado, nosso estudo apresentou uma média de idade semelhante àquela encontrada na Ilha de Curaçau ${ }^{(3)}$ de 34,6 anos.

O pico de incidência de 32,7 por $100.000 /$ ano (95\% IC 15,0-62,1) foi observado em mulheres na faixa de 35 a 39 anos, semelhantemente ao encontrado por Nossent ${ }^{(3)}$, na Ilha de Curaçau, onde o pico de incidência ocorreu na mulheres entre 15 e 44 anos. Em contraste, nos estudos realizados na Suécia ${ }^{(23)}$ e na Islândia ${ }^{(29)}$, os picos de incidência ocorrerem em mulheres com idade mais avançada (55 a 59 anos e 50 a 59 anos, respectivamente).

A mediana de duração da doença (tempo entre o início dos sintomas atribuído pelo médico à doença e o diagnóstico) de 10 meses, em nosso estudo, foi menor que a encontrada por Hopkinson et al. ${ }^{(30)}$ de 61 meses.

Em nosso estudo encontramos uma alta incidência de LES em Natal. Esta incidência, no entanto, ainda poderá estar sendo subestimada, pois é possível que tenhamos deixado de incluir algum caso diagnosticado e acompanhado em algum outro centro terciário no país. Todavia, se isso ocorreu, acreditamos ter sido fato raro, já que pacientes que procuram outros centros de referência sempre têm um médico no local de origem para o acompanhamento concomitante. 
A alta incidência de LES em Natal, região urbana de clima tropical, é um fato intrigante, que suscita maior atenção à doença, com conseqüente melhora nas condições de diagnóstico, e um estímulo para que estudos semelhantes sejam desenvolvidos nesta região. A necessidade de identificação de fatores genéticos e ambientais que possam estar associados a esta alta incidência da doença em Natal também aponta para a necessidade da realização de outros estudos nesta população e em outras regiões do Brasil.

\section{REFERÊNCIAS}

1. Siegel M, Lee SL. The epidemiology of systemic lupus erythematosus. Sem Arthritis Rheum 1973;3:1-54.

2. Nived O, Sturfelt G, Wollheim F. Systemic lupus erythematosus in a adult population in Southern Sweden: incidence, prevalence and validity of ARA revised classification criteria. Br J Rheumatol 1985; $24: 147-54$

3. Nossent JC. Systemic lupus erythematosus on the Caribbean island of Curaçao: an epidemiological investigation. Ann Rheum Dis 1992;51:1197-1201.

4. Hopkinson ND, Doherty M, Powell RJ. The prevalence and incidence of systemic lupus erythematosus in Nottingham, UK, 1989-1990. Br J Rheumatol 1993;32:110-5.

5. Fessel WJ. Systemic lupus erythematosus in the community: Incidence, prevalence, outcome, and first symptoms; the high prevalence in black women. Arch Intern Med 1974;134:1027-35.

6. Michet CJ Jr, McKenna CH, Elveback LR, Kaslow RA, Kurland LT. Epidemiology of systemic lupus erythematosus and other connective tissue diseases in Rochester, Minnesota, 1950 through 1979. Mayo Clin Proc 1985;60:105-13.

7. McCarty DJ, Manzi S, Medsger TA Jr., Ramsey-Goldman R, LaPorte RE, Kwoh CK. Incidence of systemic lupus erythematosus. Race and gender differences. Arthritis Rheum 1995;38:1260-70.

8. Hochberg MC. The incidence of systemic erythematosus lupus in Baltimore, Maryland, 1970-1977. Arthritis Rheum 1985;28:80-6.

9. Johnson AE, Cavalcanti FS, Gordon C, et al. Cross sectional analysis of patients with systemic lupus erythematosus in England, Brazil and Sweden. Lupus 1994;3:501-6.

10. Kurland LT, Hauser WA, Ferguson RH, Holley KE. Epidemiologic features of diffuse connective disorders in Rochester, Minn., 1951 through 1967, with special reference to Systemic Lupus Erythematosus. Mayo Clin Proc 1969;44:649-63.

11. Sanchez-Guerrero J, Karlson EW, Liang MH, Hunter DJ, Colditz GA. Past use of oral contraceptives and the risk for development systemic lupus erythematosus. Arthritis Rheum 1997;40:804-8.

12. Sanchez-Guerrero J, Liang MH, Karlson EW, Hunter DJ, Colditz GA. Postmenopausal estrogen therapy and the risk for development systemic lupus erythematosus. Ann Intern Med 1995;122:430-1.

13. Zamanski GB. Sunlight-induced pathogenesis in systemic lupus erythematosus. J Invest Dermatol 1985;85:179-80.

14. Petzelbauer P, Binder M, Nikolakis P, Ortel B, Honigsmann H. Severe sun sensitivity and the presence of antinuclear antibodies in patients with polymorphous light eruption-like lesions. J Am Acad Dermatol 1992;26:68-74.

\section{AGRADECIMENTOS}

Os autores gentilmente agradecem o Dr. Luis Eduardo Coelho Andrade e a Dra. Rozana Mesquita Ciconelli, da UNIFESP, pela organização do Projeto Pronuclear, a professora Maria do Rosário Dias de O. Latorre e Denise P. Bergamaschi, da USP, pela análise estatística, e os colegas médicos que trabalham em Natal, RN.

15. Marques Neto JF, Gonçalves HT, Langen LFOB, et al. Estudo multicêntrico da prevalência da artrite reumatóide do adulto em amostras da população brasileira. Rev Bras Reumatol 1993;33:169-73.

16. Censo 2000 (Dados preliminares). Instituto Brasileiro de Geografia e Estatística (IBGE) (www.ibge.gov.br)

17. Long CS, Miller AJ, Lee HT, Wild JD, Przywarty RC, Hufford D. Ultraviolet index forecasts issued by the National Weather Service. Bull Am Meteorological Soc 1996;77:729-48.

18. Kirchhoff VWJH. Medidas recentes de UV-B. In: Ozônio e radiação UV-B,1.ㄹ ed, São José dos Campos, Transtec Editorial, 1995.

19. Tan E, Cohen AS, Fries JF, et al. The 1982 revised criteria for the classification of systemic erythematosus lupus. Arthritis Rheum 1996;25:1271-77.

20. Censo 1991. Instituto Brasileiro de Geografia e Estatística (IBGE) (www.ibge.gov.br)

21. Censo 1996 (Contagem populacional). Instituto Brasileiro de Geografia e Estatística (IBGE) (www.ibge.gov.br)

22. Uramoto KM, Michet CJ Jr., Thumboo J, Sunku J, O'Fallon WM, Gabriel SE. Trends in the incidence and mortality of systemic lupus erythematosus, 1950-1992. Arthritis Rheum 1999;42:46-50.

23. Jonsson H, Nived O, Sturfelt G, Silman A. Estimating the incidence of systemic lupus erythematosus in a defined population using multiple sources of retrieval. Br J Rheumatol 1990;29:185-8.

24. Stahl-Hallengren C, Jonsen A, Nived O, Sturfelt G. Incidence studies of systemic lupus erythematosus in Southern Sweden: increasing age, decreasing frequency of renal manifestations and good prognosis. J Rheumatol 2000;27:685-91.

25. Ribeiro D. O povo brasileiro: a formação e o sentido do Brasil, $2 .^{a}$ ed, São Paulo, Companhia das Letras, 1995.

26. Cascudo LC. História do Rio Grande do Norte, $1 .^{a}$ ed, Rio de Janeiro, Ministério da Educação e Cultura, 1955.

27. Johnson AE, Gordon C, Palmer RG, Bacon PA. The prevalence and incidence of systemic lupus erythematosus in Birmingham, England. Relationship to ethnicity and country of birth. Arthritis Rheum 1995;38:551-8.

28. Jonsson H, Nived O, Sturfelt G. Outcome in systemic lupus erythematosus. A prospective study of patients from a defined population. Medicine (Baltimore) 1989;8:141-50.

29. Gudmundsson S, Steinsson K. Systemic lupus erythematosus in Iceland 1975 through 1984. A nationwide epidemiological study in a unselected population. J Rheumatol 1990;17:1162-7.

30. Hopkinson ND, Doherty M, Powell RJ. Clinical features and racespecific incidence/prevalence rates of systemic lupus erythematosus in a geographically complete cohort of patients. Ann Rheum Dis 1994;53:675-80. 